\title{
Evaluación a largo plazo del estado nutricional, composición corporal y densidad mineral ósea en mujeres operadas de bypass gástrico: impacto del nivel socioeconómico
}

\author{
María Pía de la Maza, Laura Leivaa, G ladys Barrerab, \\ Carolina Boggiano, Tomás Herrerac, Yanet Pérez ${ }^{\mathrm{d}}$, \\ Vivien G attás ${ }^{d}$, Daniel Bunout, Sandra Hirsch \\ Nutritional status, body composition \\ and bone mineral density in gastric \\ bypass females: \\ impact of socioeconomic level
}

Background: Roux-en-Y gastric bypass (RYGBP) has had a positive impact on co-morbidities associated with obesity. However, in the long-term it can induce micronutrient deficiencies. Aim: To perform a complete nutritional assessment in a group of women previously operated of RYGBP, from different socioeconomic levels (SEL). Patients and Methods: Thirty three women (19 high SEL and 14 low SEL), were assessed by dietary recalls, anthropometric measurements, muscle strength, bone mineral density, routine clinical laboratory, serum levels of vitamin B12, 25OHvitamin $\mathrm{D}$, folate, calcium, ferritine, ceruloplasmin and indicators of bone turnover (parathohormone, osteocalcin and urinary pyridinolines). Their values were compared to those of 30 control women (18 high SEL and 12 low SEL). Results: Low SEL operated women consumed fewer vitamin and mineral supplements compared with their high SEL pairs. No cases of vitamin B12, folic acid or copper deficiencies were detected. Frequency of iron deficiency was similar in patients and controls. Vitamin D insufficiency was higher among patients than in controls $(p=0,047)$, regardless SEL Patients had also a higher frequency of high serum PTH and osteocalcin and urinary pyridinoline levels. However, no differences in bone mineral density were observed between operated women and controls. Conclusions: Vitamin and mineral deficiencies were lower than expected among operated women. However, problems associated with vitamin D deficiency were highly prevalent among patients operated of RYGBP, irrespective SEL These alterations were only detectable through specific markers at this stage, because they did not translate into lower bone mineral density (BMD) of surgical patients, probably due to the higher pre-operative BMD of these morbid obese patients (Rev Méd Chile 2008; 136: 1415-23).

(Key w ords: Gastric bypass; Nutritional status; Socioeconomic factors)

Recibido el 10 de abril, 2008. Aceptado el 28 de julio, 2008.

Trabajo financiado por Proyecto MULT 05/23-2, DI Universidad de Chile.

Instituto de Nutrición y Tecnología de los Alimentos (INTA), Universidad de Chile.

aTecnóloga Médica

${ }^{b}$ Enfermera Universitaria

cProfesor Educación Física

d Nutricionista

Correspondencia a: Dra. M Pía de la Maza. Macul 5540,

Santiago. Fax: 2214030. E mail: mpmaza@inta.cl 
$\mathrm{E}^{1}$ bypass gástrico en Y de Roux (BPGY) ha tenido impacto favorable en el tratamiento de la obesidad mórbida, por cuanto permite mayores reducciones de peso que el tratamiento médico, con mantención del peso reducido ${ }^{1}$ y adecuado control de las comorbilidades ${ }^{2,3}$. Recientemente se han demostrado sus beneficios en términos de mortalidad a 10 años ${ }^{4}$.

Esta cirugía reduce el volumen de los alimentos ingenidos y limita la digestión y absorción de los nutrientes en el intestino, pero puede inducir carencias nutricionales, especialmente sobre micronutrientes que se absorben en el duodeno y yeyuno proximal (hierro, calcio, vitamina $\mathrm{D}$, folato, zinc y cobre), 0 requieren la secreción de ácido y factor intrínseco (vitamina B12) $^{5}$. Se han informado deficiencias de vitaminas del complejo B con manifestaciones neurológicas $^{6-8}$ y anemia megaloblástica ${ }^{9}$, alopecia por carencia de zinc ${ }^{5}$, anemia ferropénica ${ }^{9-11}$, además de trastomos del metabolismo óseo ${ }^{12,13}$. La hipovitaminosis A y déficit proteico son menos frecuentes ${ }^{14,15}$. A pesar de estas evidencias, y a la normativa ministerall $^{16}$, no se ha estandanizado la suplementación postoperatoria, especialmente al largo plazo, donde muchos pacientes abandonan los controles médicos.

Por otra parte, la pobreza se asocia a mayor prevalencia y severidad de la obesidad, pero un menor acceso al sistema de salud y medios para lograr un estado nutricional saludable. Entre los pacientes con BPGY, los menos adherentes y vomitadores son los que presentan complicaciones neurológicas por hipovitaminosis ${ }^{17,18}$, parte de ello podría atribuirse al menor acceso a controles médicos. Otras publicaciones han informado mortalidad mayor a la esperable en pacientes de mayor edad y nivel socioeconómico (NSE) bajo ${ }^{18,19}$.

El objetivo del presente estudio fue evaluar a largo plazo el estado nutricional, la suplementación nutricional, la composición corporal y la densidad mineral ósea (DMO) de mujeres operadas de BPGY un año y medio antes o más, y relacionarlos con el NSE.

\section{PACIENTES Y MÉTOdOS}

Pacientes. Luego de firmar un consentimiento informado, seleccionamos 33 mujeres, con un peńodo postoperatorio de BPGY igual o superior a 1 año y medio y 30 mujeres sin antecedentes de cirugía bariátrica, con promedio de edad e IMC similar, como controles. Ambos grupos fueron divididos en 2 categonías según NSE, en base a una encuesta de la unidad de Asistencia Social del Servicio de Salud Metropolitano Sur, de Santiago. Esta encuesta registra nivel educacional, tipo de trabajo, estabilidad laboral e ingreso mensual del grupo familiar. Según la clasificación del Instituto Consultor en Comercialización y Mercado (ICCOM*), se consideró NSE alto a los estratos $\mathrm{ABC1}$ y C2 y NSE bajo a C3, D y E. La técnica quirúrgica fue bypass abierto en 24 casos (4 con gastrectomía total) o laparoscópico en 9 casos. Las mujeres de NSE alto fueron operadas en el Hospital Clínico de la Pontificia Universidad Católica (8 pacientes), Hospital Clínico de la Universidad de Chile (5 pacientes), Clínica Indisa (3 pacientes), Hospital Bamos Luco-Trudeau (1 paciente), Clínica Dávila (1 paciente) y Hospital Militar (1 paciente). Las pacientes de NSE bajo fueron operadas en el Hospital Barros Luco Trudeau (11 pacientes) y Hospital Clínico de la Universidad de Chile (3 pacientes).

Historia clínica y encuesta alimentaria. En cada paciente se efectuó una historia clínica completa, registrando los cambios de peso desde el preoperatorio hasta el momento actual, la tolerancia alimentaria como buena, regular o mala (según dispepsia, vómitos, diarrea y dumping asociados a alimentación), la ingesta calórico-proteica (por encuestas de tipo recordatorio de $24 \mathrm{~h}$ y tendencia de consumo ${ }^{20,21}$ ), el consumo de suplementos vitamínicos durante el primer año y al momento de la evaluación y el número de controles médicos y nutricionales a los que asistió durante el primer año postoperatorio.

Evaluación nutricional y composición corporal. Se efectuaron mediciones antropométricas (IMC, circunferencias de cintura y cadera), densitometría radiológica (DEXA) para medir composición corporal y masa ósea, en un equipo Lunar Prodigy Encore 2007 (software version 11,30,062). Se midió fuerza muscular en una mesa de cuádriceps adosada a un transductor de presión, fuerza de agarre de la mano con un dinamómetro (Therapeutic Instruments, Clifton, NJ, EE.UU.) y se tomó

*www.iccom.cl 
muestras de sangre y orina para laboratorio clínico (hemograma, glicemia, insulina, creatininemia, pruebas hepáticas, TSH y perfil lipídico) en el Laboratorio Vidaintegra, Santiago de Chile.

Estado nutricional de vitaminas y minerales: Entre los micronutrientes séricos se determinó ferritina, calcio total, magnesio (con metodología automatizada en el Laboratorio Vidaintegra), actividad de ceruloplasmina (a través del método colorimétrico de Ravin), vitamina B12 y ácido fólico (con radioinmunoensayo simultáneo en fase sólida Dualcount, DPC Diagnostic Products Corporation CA, USA) y 25-OH vitamina D (mediante radioinmunoensayo, DiaSorin USA).

Evaluación del metabolismo óseo: Como indicadores de recambio óseo, se determinó niveles séricos de las moléculas intactas de paratohormona (iPTH), osteocalcina y deoxipiridinolinas urinarias (Pyrilinks D), mediante inmunoensayo quimioluminiscente (IMMULTE 1000, Siemens Medical Solution Diagnostics, UK). Las muestras fueron obtenidas entre abril y noviembre.

El grupo control fue sometido a los mismos procedimientos que las pacientes operadas. El estudio fue aprobado por el comité de ética del INTA de acuerdo a la declaración de Helsinki.

Estadística. Los resultados fueron analizados en el programa estadístico Stata para Windows versión
8.0. Los datos se presentan como promedio \pm DS, y se calculó test de Fischer para comparar proporciones y análisis de varianza de una vía para analizar diferencias entre los 4 grupos.

\section{RESUlTADOS}

Entre las pacientes operadas de BPGY, 19 correspondían a NSE alto y 14 al NSE bajo (las características generales antropométricas se presentan en la Tabla 1). El período postoperatorio promedio fue de 42 meses (17 a 94 meses), siendo éste menor a 2 años en sólo 4 casos. La baja de peso en el período estudiado promedió $41 \pm 11$ $\mathrm{kg}$. En el grupo control, 18 pertenecían al NSE alto (una de las cuales fue excluida del análisis por antecedentes de enfermedad celíaca) y 12 al NSE bajo, con edad promedio de $41 \pm 9$ años.

En cuanto a tolerancia alimentaria, en 6 pacientes fue regular ( 3 de NSE alto y 3 de NSE bajo). Los alimentos mal tolerados (cames rojas, fideos, arroz) correspondían a los mencionados habitualmente por pacientes operados de BPGY, pero solamente en 2 pacientes coincidía con una alta frecuencia de vómitos y diarrea (3 y 2 veces por semana, respectivamente). Dieciséis casos informaron persistencia de dumping, aunque en forma muy ocasional.

Tabla 1. Variables antropométricas en pacientes operadas versus control

\begin{tabular}{|lccccc|}
\hline & \multicolumn{2}{c}{ Bariátricas } & \multicolumn{2}{c|}{ Controles } & Anova \\
& NSE alto $(\mathbf{n}=\mathbf{1 9})$ & NSE bajo $(\mathbf{n}=\mathbf{1 4})$ & NSE alto $(\mathbf{n}=\mathbf{1 7})$ & NSE bajo ( $\mathbf{n}=\mathbf{1 2})$ & $\mathbf{p}$ \\
\hline Edad (años) & $44 \pm 9$ & $45 \pm 9$ & $43 \pm 10$ & $40 \pm 7$ & 0,34 \\
Peso preoperatorio $(\mathrm{kg})$ & $110 \pm 15$ & $123 \pm 14$ & & & 0,015 \\
IMC preoperatorio $\left(\mathrm{kg} / \mathrm{m}^{2}\right)$ & $43 \pm 5$ & $49 \pm 4$ & & & 0,0003 \\
& & & & & \\
Peso actual $(\mathrm{kg})$ & $71 \pm 11$ & $82 \pm 10^{\mathrm{a}}$ & $65 \pm 10$ & $76 \pm 14$ & 0,0009 \\
Talla $(\mathrm{cm})$ & $161 \pm 5$ & $159 \pm 8$ & $162 \pm 7$ & $155 \pm 5$ & 0,279 \\
IMC $\left(\mathrm{kg} / \mathrm{m}^{2}\right)$ & $27 \pm 4^{\mathrm{c}}$ & $32 \pm 4^{\mathrm{a}}$ & $25 \pm 4^{\mathrm{b}}$ & $31 \pm 6$ & 0,0001 \\
Cintura $(\mathrm{cm})$ & $85 \pm 10$ & $93 \pm 13^{\mathrm{a}}$ & $82 \pm 8$ & $90 \pm 11$ & 0,0343 \\
Cadera $(\mathrm{cm})$ & $101 \pm 9$ & $109 \pm 10^{\mathrm{a}}$ & $99 \pm 9$ & $102 \pm 10$ & 0,0416 \\
\hline
\end{tabular}

IMC: Indice de masa corporal. Significativo si $p<0,05$ - Análisis post hoc de Bonferroni:

${ }^{\text {a }}$ Diferente de controles de NSE alto

${ }^{\mathrm{b}}$ Diferente de controles de NSE bajo

${ }^{\mathrm{c}}$ Diferente de bariátricas de NSE bajo 
Durante el primer año de postoperatorio, las pacientes fueron controladas alrededor de 6 veces por el cirujano. El número de visitas a nutricionista o nutriólogo fue variable, dependiendo del NSE. En el nivel alto 4,8 \pm 5 , mientras que en el NSE bajo 2,8 \pm 2 visitas ( $p=0,07$ ); 6 pacientes no habían sido controladas nunca desde el punto de vista nutricional. El consumo de suplementos de vitaminas o minerales, tanto durante los primeros meses de la cirugía como al momento de la evaluación, resultó extremadamente variable en cuanto a productos y dosis indicada. Al momento de la evaluación se constató que no estaban recibiendo suplementación alguna 8 de 14 pacientes de NSE bajo, versus 3 de 19 pacientes del NSE alto $(p=0,02)$. Dos pacientes de NSE bajo no habían recibido suplementación alguna de vitaminas o minerales durante todo el período postoperatorio, excepto por 3 dosis de vitaminas B1, B6 y B12 intramuscular en una de ellas.

Las pacientes operadas de NSE alto consumían $1.454 \pm 636$ Kcals y $57 \pm 16$ g proteínas, y aquellas de NSE bajo $1.536 \pm 475$ Kcal y $60 \pm 25$ g proteínas ( $p=0,67$ y 0,59 , respectivamente). El consumo de fibra dietaria era marginalmente superior en las pacientes operadas de NSE alto $(8,5 \pm 6 \mathrm{~g}$ versus $5,2 \pm 3 \mathrm{~g} /$ día, respectivamente, $p=0,072$ ). Este patrón se repitió entre las mujeres del grupo control, es decir similar aporte calórico (1.637 \pm 520 versus $1.794 \pm 591 \mathrm{Kcal} /$ día) y proteico (64 \pm 18 versus $60 \pm 18 \mathrm{~g} /$ día), pero con mayor aporte de fibra dietaria entre mujeres de NSE alto $(12,9 \pm$ 5 versus $5,3 \pm 2 \mathrm{~g} /$ día, $\mathrm{p}<0,001$ ). El consumo de calcio promedió $750 \mathrm{mg} /$ día, sin diferencias entre los 4 grupos.

En las Tablas 1 a 3 se muestran las comparaciones de las variables antropométricas, fuerza muscular y densitometría entre los 4 grupos estudiados y en las Tablas 4 y 5 los resultados de los análisis de laboratorio. Las pruebas hepáticas resultaron normales, con excepción de una paciente operada de NSE alto con antecedente de ingesta alcohólica excesiva que presentó una leve alteración. Asimismo, los niveles de magnesio y calcio sérico total estuvieron dentro de los rangos normales.

$\mathrm{Si}$ bien en promedio los micronutrientes séricos se encontraron dentro de los rangos normales, se detectó déficit de hierro (ferritina $<13 \mathrm{ug} / \mathrm{dL}$ ) en 17 de las 33 pacientes operadas versus 9 de 30 controles $(p=0,12)$ y anemia en 9 pacientes operadas versus 3 controles ( $p=0,11)$, sin diferencias entre NSE. En cuanto a 25-OH vitamina D, 13 pacientes versus 5 controles tenían niveles $<16$

Tabla 2. Composición corporal (DEXA) y fuerza muscular en pacientes operadas versus controles

\begin{tabular}{|c|c|c|c|c|c|}
\hline & \multicolumn{2}{|c|}{ Bariátricas } & \multicolumn{2}{|c|}{ Controles } & \multirow{2}{*}{$\begin{array}{c}\text { Anova } \\
p\end{array}$} \\
\hline & NSE alto ( $n=19$ ) & NSE bajo ( $\mathrm{n}=14$ ) & NSE alto ( $n=17$ ) & NSE bajo ( $n=12$ ) & \\
\hline Masa grasa $(\mathrm{kg})$ & $28 \pm 7$ & $37 \pm 9^{a}$ & $26 \pm 12$ & $33 \pm 9$ & 0,0074 \\
\hline Masa grasa $(\%)$ & $39 \pm 6$ & $42 \pm 6$ & $35 \pm 8^{b c}$ & $43 \pm 5$ & 0,0030 \\
\hline Masa libre de grasa (kg) & $40 \pm 4$ & $44 \pm 6$ & $39 \pm 4$ & $40 \pm 5$ & 0,0639 \\
\hline Masa libre de grasa (\%) & $61 \pm 6$ & $58 \pm 6$ & $65 \pm 8^{\mathrm{bc}}$ & $57 \pm 5$ & 0,0030 \\
\hline FM cuádriceps derecho (kg) & $36 \pm 9$ & $32 \pm 10$ & $44 \pm 8^{b c d}$ & $34 \pm 6$ & 0,0012 \\
\hline FM cuádriceps izquierdo $(\mathrm{kg})$ & $35 \pm 7$ & $32 \pm 9$ & $46 \pm 10^{\mathrm{bcd}}$ & $38 \pm 6$ & 0,0001 \\
\hline FM mano derecha $(\mathrm{kg})$ & $28 \pm 4$ & $26 \pm 6$ & $31 \pm 5 b$ & $26 \pm 4$ & 0,0224 \\
\hline FM mano izquierda $(\mathrm{kg})$ & $26 \pm 4$ & $25 \pm 6$ & $28 \pm 3$ & $24 \pm 3$ & 0,1085 \\
\hline
\end{tabular}

FM: Fuerza muscular. Significativo si p $<0,05$ - Análisis post hoc de Bonfermoni:

${ }^{a}$ Diferente de controles de NSE alto

${ }^{b}$ Diferente de controles de NSE bajo

${ }^{\mathrm{c}}$ Diferente de bariátricas de NSE bajo

${ }^{\mathrm{d}}$ Diferente a bariátricas de NSE alto 
Tabla 3. D ensidad y contenido mineral óseo (D EXA) en pacientes versus controles

\begin{tabular}{|c|c|c|c|c|c|}
\hline & \multicolumn{2}{|c|}{ Bariátricas } & \multicolumn{2}{|c|}{ Controles } & \multirow{2}{*}{$\begin{array}{c}\text { Anova } \\
p\end{array}$} \\
\hline & NSE alto ( $n=19$ ) & NSE bajo ( $n=14$ ) & NSE alto ( $n=17$ ) & NSE bajo ( $n=12$ ) & \\
\hline CMO total $(\mathrm{g})$ & $2.713 \pm 415$ & $2.763 \pm 391$ & $2.558 \pm 415$ & $2.455 \pm 274$ & 0,156 \\
\hline DMO total $\left(\mathrm{g} / \mathrm{cm}^{2}\right)$ & $1,19 \pm 0,09$ & $1,21 \pm 0,08$ & $1,18 \pm 0,1$ & $1,18 \pm 0,1$ & 0,789 \\
\hline T score total (DS) & $0,88 \pm 1,2$ & $1,01 \pm 1,0$ & $0,66 \pm 1,2$ & $0,64 \pm 1,2$ & 0,801 \\
\hline CMO cuello femoral (g) & $5,04 \pm 0,9$ & $5,2 \pm 0,8$ & $4,7 \pm 0,8$ & $5,3 \pm 0,7$ & 0,188 \\
\hline DMO cuello femoral $\left(\mathrm{g} / \mathrm{cm}^{2}\right)$ & $1,07 \pm 0,2$ & $1,09 \pm 0,2$ & $0,97 \pm 0,2$ & $1,14 \pm 0,2$ & 0,085 \\
\hline T-score cuello femoral (DS) & $0,76 \pm 1,4$ & $0,88 \pm 1,5$ & $-0,09 \pm 1,3$ & $1,3 \pm 1,5$ & 0,091 \\
\hline Frecuencia osteopenia (n) & 1 & 1 & 4 & 1 & \\
\hline CMO columna L2-L4 (g) & $54,0 \pm 12$ & $51,7 \pm 9$ & $53,5 \pm 14$ & $49,4 \pm 8$ & 0,782 \\
\hline DMO columna L2-L4 $\left(\mathrm{g} / \mathrm{cm}^{2}\right)$ & $1,3 \pm 0,2$ & $1,3 \pm 0,1$ & $1,3 \pm 0,2$ & $1,2 \pm 0,1$ & 0,824 \\
\hline T-score columna L2-L4 (DS) & $0,96 \pm 1,5$ & $0,45 \pm 1,2$ & $0,65 \pm 1,8$ & $0,38 \pm 1,2$ & 0,723 \\
\hline Frecuencia osteopenia (n) & 2 & 2 & 4 & 2 & \\
\hline
\end{tabular}

$\mathrm{CMO}=$ Contenido mineral óseo. DMO =densidad mineral ósea

Osteopenia =T-score $-1,0$ a $-2,5$ DS, Osteoporosis $=T$-score $<2,5$ DS

Tabla 4. Exámenes de laboratorio clínico en pacientes versus controles

\begin{tabular}{|lccccc|}
\hline $\begin{array}{l}\text { Niveles séricos } \\
\text { (unidades) [rango normal] }\end{array}$ & \multicolumn{2}{c}{ Bariátricas } & \multicolumn{2}{c|}{ Controles } & Anova \\
& NSE alto (n=19) & NSE bajo (n =14) & NSE alto ( $\mathbf{n = 1 7 )}$ & NSE bajo ( $\mathbf{n = 1 2 )}$ & $\mathbf{p}$ \\
\hline Hemoglobina (g/Dl) [12-16] & $12,5 \pm 2$ & $12,3 \pm 2^{\mathrm{a}}$ & $13,8 \pm 1$ & $13,1 \pm 0,8$ & 0,024 \\
CHCM (\%) [32-36] & $32,3 \pm 1,6$ & $31,9 \pm 2,1^{\mathrm{a}}$ & $33,3 \pm 0,6$ & $32,8 \pm 0,8$ & 0,040 \\
Leucocitos x 103 $\mu \mathrm{LL}[4,5-11]$ & $5,5 \pm 1,7$ & $5,8 \pm 1,2$ & $6,1 \pm 1,7$ & $6,8 \pm 1,5$ & 0,137 \\
Glicemia (mg/dL) [60-100] & $82,3 \pm 7$ & $86,7 \pm 15$ & $83,7 \pm 11$ & $88,9 \pm 12$ & 0,390 \\
Insulina (U/dL) [<12] & $5,0 \pm 2,8$ & $4,7 \pm 3,0$ & $6,1 \pm 3,7$ & $10,8 \pm 7,7$ ad & 0,003 \\
Creatinina (mg/dL) [0,5-0,9] & $0,68 \pm 0,12$ & $0,66 \pm 0,11$ & $0,75 \pm 0,8$ & $0,66 \pm 0,11$ & 0,089 \\
Albúmina (g/dL) [3,4-4,8] & $4,5 \pm 0,2$ & $4,4 \pm 0,3$ & $4,6 \pm 0,3$ & $4,4 \pm 0,3$ & 0,398 \\
Colesterol total (mg/dL) [<200] & $181 \pm 29$ & $172 \pm 33$ & $195 \pm 39$ & $185 \pm 33$ & 0,310 \\
Colesterol HDl (mg/dL) [>40] & $71 \pm 24$ & $63 \pm 9$ & $67 \pm 16$ & $54 \pm 13$ & 0,084 \\
Colesterol LDL (mg/dL) [<130] & $88 \pm 24$ & $88 \pm 28$ & $111 \pm 37$ & $107 \pm 30$ & 0,060 \\
Triglicéridos (mg/dL) [<150] & $91 \pm 36$ & $106 \pm 33$ & $87 \pm 33$ & $116 \pm 59$ & 0,204 \\
Calcio (mg/dL) [8,4-10,2] & $9,4 \pm 0,7$ & $9,1 \pm 0,3$ & $9,1 \pm 0,4$ & $9,0 \pm 0,2$ & 0,104 \\
Fósforo (g/dL) [2,7-4,5] & $3,9 \pm 0,6^{*}$ & $3,9 \pm 0,7$ & $3,4 \pm 0,5$ & $3,5 \pm 0,6$ & 0,018 \\
Magnesio (mEq/L) [1,4-2,3] & $1,7 \pm 0,2$ & $1,7 \pm 0,2$ & $1,6 \pm 0,1$ & $1,6 \pm 0,1$ & 0,111 \\
\hline
\end{tabular}

CHCM = Concentración de hemoglobina corpuscular media Significativo si $\mathrm{p} \leq 0,05$ - Análisis post hoc de Bonferroni:

a Diferente de controles de NSE alto

d Diferente a bariátricas de NSE alto

$\mathrm{ng} / \mathrm{mL}$ o $40 \mathrm{nmol} / \mathrm{L}(\mathrm{p}=0,047)$, sin diferencias entre NSE. En concordancia con ello, se detectó elevación de iPTH $(>67 \mathrm{pg} / \mathrm{mL})$ en 13 pacientes operadas versus 2 controles $(p<0,01)$ y de osteocalcina $(>13,7 \mathrm{ng} / \mathrm{mL})$ en 20 pacientes y 1 mujer del grupo control ( $p<0,001)$. La excreción 
Tabla 5. N iveles séricos de micronutrientes en pacientes versus controles

\begin{tabular}{|c|c|c|c|c|c|}
\hline \multirow{2}{*}{$\begin{array}{l}\text { Niveles séricos } \\
\text { (unidades) [rango normal] }\end{array}$} & \multicolumn{2}{|c|}{ Bariátricas } & \multicolumn{2}{|c|}{ Controles } & \multirow{2}{*}{$\begin{array}{l}\text { Anova } \\
p\end{array}$} \\
\hline & NSE alto ( $n=19$ ) & NSE bajo ( $n=14$ ) & NSE alto ( $n=17$ ) & NSE bajo ( $n=12$ ) & \\
\hline Ceruloplasmina (\%) [15-40] & $27,2 \pm 8$ & $30,9 \pm 10$ & $23,5 \pm 6^{b}$ & $32,9 \pm 8$ & 0,014 \\
\hline Ferritina (ug/L) [13-150] & $33 \pm 43$ & $43 \pm 57$ & $54 \pm 41$ & $42 \pm 33$ & 0,556 \\
\hline Folato (ng/mL) [3-17] & $22,9 \pm 6^{a}$ & $19,6 \pm 4$ & $15,8 \pm 4$ & $20,1 \pm 3$ & 0,0005 \\
\hline Vitamina B12 (pg/mL) [174-878] & $444 \pm 159$ & $439 \pm 234$ & $594 \pm 199$ & $287 \pm 91^{\mathrm{a}}$ & 0,0005 \\
\hline 25-OH vitamina $\mathrm{D}(\mathrm{ng} / \mathrm{mL})[<16]^{*}$ & $17,4 \pm 10$ & $22,8 \pm 10$ & $22,3 \pm 9$ & $18,8 \pm 6$ & 0,318 \\
\hline iPTH (pg/mL) [11-67] & $68,6 \pm 23$ & $68,8 \pm 25$ & $58,9 \pm 14$ & $50,1 \pm 15$ & 0,040 \\
\hline Osteocalcina (ng/mL) [3,1-13,7] & $17,7 \pm 9,5$ & $18,5 \pm 8,3$ & $6,4 \pm 4,1^{\mathrm{cd}}$ & $7,2 \pm 2,6^{\mathrm{cd}}$ & $<0,0001$ \\
\hline Pyrilinks (nmol/molCreat) & $10,5 \pm 3,9$ & $10,1 \pm 3,7$ & $6,2 \pm 1,1_{\mathrm{cd}}$ & $5,5 \pm 0,6 * *$ & 0,0011 \\
\hline
\end{tabular}

Significativo si p <0,05 - Análisis post hoc de Bonferroni:

a Diferente de controles de NSE alto

${ }^{\mathrm{b}}$ Diferente de controles de NSE bajo

${ }^{\mathrm{c}}$ Diferente de bariátricas de NSE bajo

d Diferente a bariátricas de NSE alto

* Punto de corte $<16 \mathrm{ng} / \mathrm{mL}$ por razones expuestas en el texto

${ }^{*}$ Datos promedio de 2 mujeres

de piridinolinas urinarias también resultó significativamente más alta en pacientes operadas versus controles. No se detectó ningún caso de déficit de vitamina B12, folato, ni cobre.

\section{Discusión}

En este estudio comprobamos que, a largo plazo, la cirugía bariátrica permite que pacientes previamente obesas mórbidas se acerquen a la población general en términos de peso y de variables de laboratorio clínico. Sin embargo, también observamos que la pacientes operadas, especialmente las de NSE bajo, quedaron con IMC en rango de obesidad y con porcentaje de grasa superior a $40 \%$; en cuanto a su masa libre de grasa (MLG), en términos absolutos no se encontró disminuida, siendo comparable a las mujeres control de NSE bajo. Sin embargo, las mujeres control de NSE alto presentaron una proporción mayor de MLG y menor densidad mineral ósea, en concordancia con un peso significativamente menor, pero a pesar de ello, tenían una fuerza muscular significativamente superior comparado con las mujeres de NSE bajo (controles y operadas). Probablemen- te esto guarda relación con mejores hábitos de ejercicio, dato que no pudimos objetivar, o con su mejor estatus de vitamina $\mathrm{D}^{22}$.

Adicionalmente comprobamos que la incidencia de deficiencias de vitaminas y minerales era menor a lo publicado ${ }^{23}$, aun considerando que un porcentaje de estas pacientes prácticamente no habían recibido suplementación. En el caso de ácido fólico ello puede atribuirse a la fortificación de la harina a nivel nacional, iniciada en el año $2000^{24}$, pero para el caso de la vitamina B12 demostramos que la fortificación exclusivamente con folato podría enmascarar su déficit en adultos mayores ${ }^{25}$, por lo cual hubiésemos esperado más casos de carencia de esta vitamina.

El resultado más relevante de este estudio lo constituyen las alteraciones del metabolismo óseo entre las mujeres operadas. Esto ha sido previamente informado ${ }^{12,26}$ y parece depender, por una parte, de un déficit de calcio asociado a menor ingestión de lácteos o a una menor absorción por el bypass duodeno-yeyunal o por carencia de vitamina D. Dado que el BPGY en general no induce en forma relevante malabsonción de grasas y vitaminas liposolubles, como otras técnicas quirúrgicas, no se ha aclarado totalmente las causas 
del déficit de vitamina $\mathrm{D}$ entre pacientes bariátricos, ni se conoce su riesgo de fracturas a largo plazo. $\mathrm{Si}$ bien no existe consenso en cuanto a concentraciones séricas adecuadas, el déficit de vitamina $\mathrm{D}$ es altamente prevalente en la población general y es aún mayor en obesos mórbidos antes y después de la cirugía 27,28 , independiente de la exposición solar ${ }^{29}$. Más aún, las alteraciones óseo-metabólicas no responden a las dosis de suplementación habituales de calcio y vitamina $\mathrm{D}^{13}$.

Si bien se consideran óptimos niveles de 25 $\mathrm{OH}$ vitamina D superiores a $30-32 \mathrm{ng} / \mathrm{mL}$ (75-80 $\mathrm{nmol} / \mathrm{L})$ y deficiencia como niveles inferiores a 8 $\mathrm{ng} / \mathrm{mL}$ o $20 \mathrm{nmol} / \mathrm{L}$, los niveles intermedios (insuficiencia) se asocian a osteoporosis ${ }^{30}$. En este estudio utilizamos $16 \mathrm{ng} / \mathrm{mL}$ (40 nmol/L) como punto de corte, debido a que en trabajos previos comprobamos que bajo este límite se elevan los niveles de iPTH, señalando resorción ósea ${ }^{31}$, y comprobamos que la suplementación habitual de calcio y vitamina D (400 UI colecalciferol) mejoró, pero no corrigió totalmente el déficit, en ancianos $^{32}$; lo mismo ocurre en gastrectomizados ${ }^{33,34}$. La respuesta a la suplementación en pacientes obesos sometidos a BPGY parece ser menos eficiente $^{12,13,35-37}$, motivo por el cual se ha planteado que la terapia debiera ser mucho más agresiva en situaciones de deficiencia de vitamina $\mathrm{D}$, con dosis superiores a 1.300 UI por vía oral o 400.000 UI por vía intramuscular cada 2 me$\operatorname{ses}^{30,35,36}$. Si hubiéramos establecido $30 \mathrm{ng} / \mathrm{mL}$ como límite inferior, prácticamente toda la muestra (pacientes y controles) hubiese sido considerada como deficitaria; como se aprecia en la Tabla 4, los niveles promedio de $25-\mathrm{OH}$ vitamina $\mathrm{D}$ eran cercanos al límite inferior tanto en las pacientes operadas como en el grupo control, aunque un mayor porcentaje de las pacientes bariátricas resultaron deficitarias. Sin embargo, las concentraciones de iPTH, que tienden a ser superiores entre las pacientes operadas, como las de osteocalcina, marcador de formación ósea, y los de piridinolinas urinarias, indicadoras de resorción ósea, fueron significativamente más altos entre las pacientes bariátricas, señalando un recambio óseo más activo. Es destacable que estas diferencias no se relacionaron con el NSE, por lo cual en esta muestra parecieron depender más de la cirugía que de la suplementación o ingesta dietaria. Las alteraciones del recambio óseo tampoco se vieron reflejadas en la densitometría ósea, por cuanto el contenido mineral óseo (CMO) y DMO de las pacientes operadas tendió a ser incluso superior al de las controles. Esto no es sorprendente, debido al incremento de masa ósea de los sujetos obesos. Sin embargo, en un estudio previo, al evaluar longitudinalmente pacientes operadas de BGYR que mantenían un peso estable luego de 2 años de cirugía, detectamos una disminución significativa del contenido y densidad mineral ósea, a nivel de cuello femoral (2,6\% en 1 año) (De la Maza MP, resultados no publicados), similar al de otros estudios longitudinales en BPGY $^{12,13}$ y banda gástrica ${ }^{38}$.

Además, como era de esperarse, tanto DMO como CMO eran inferiores entre las 12 mujeres postmenopáusicas (10 operadas, 2 con terapia de sustitución y 6 controles, 4 con sustitución hormonal). Sin embargo al excluirlas del análisis se mantuvieron los resultados. Por otra parte los niveles de vitamina D, iPTH y osteocalcina resultaron similares al comparar pre y postmenopáusicas.

En este estudio se intentó, además, establecer la influencia del NSE sobre los parámetros nutricionales. Como se señaló, las alteraciones del metabolismo óseo no se vieron afectadas por el NSE. Sin embargo, la dificultad en el acceso a la salud entre los grupos más pobres se manifestó claramente en un menor número de controles nutricionales e insuficiente suplementación de micronutrientes entre las pacientes provenientes del NSE bajo. Sorprendentemente esto no se vio reflejado en menores niveles de las vitaminas del complejo B analizadas, aunque no podemos asegurar que no exista déficit de otras vitaminas. En cuanto a los minerales analizados, la actividad de ceruloplasmina, indicador del estado de cobre, resultó normal en $100 \%$ de los casos y la frecuencia de déficit de hierro, mineral más frecuentemente deficitario entre pacientes operados de BPGY, resultó prácticamente igual entre pacientes y controles de diferente NSE.

Dentro de las limitaciones de este estudio está el reducido tamaño muestral y la ausencia de datos preoperatorios, que permitinan efectuar análisis longitudinales de la evolución de las variables nutricionales, por cuanto existen datos de una prevalencia importante de carencias de micronutrientes en pacientes obesos mórbidos, incluso antes de la cirugía ${ }^{39}$. Sin embargo, ésta es la 
realidad con que nos enfrentamos: en esta muestra comprobamos que en la mayor parte de los pacientes no se habían efectuado evaluaciones nutricionales exhaustivas en ningún momento de la evolución, el acceso a nutricionistas o médicos especialistas en nutrición fue limitado, y la suplementación de micronutrientes no pareció guardar relación con los especiales requerimientos de estos

\section{REFERENCIAS}

1. Awad W, Aray A, Martínez C, Oñate V, Turu V, YARMUCH J. Descenso ponderal y calidad de vida mediante la cirugía de bypass gástrico con y sin anillo de calibración. Descenso ponderal y calidad de vida mediante la cirugía de bypass gástrico. Rev Chil Cir 2008; 0: 17-21.

2. BROLN RE. Bariatric surgery and long-term control of morbid obesity. JAMA 2007; 288: 2793-6.

3. Papapietro K, Díaz E, Csendes A, Díaz Jc, Braghetto I, BuRdiles P ET AL. Efectos de bypass gástrico en peso corporal, glicemia, lípidos séricos y presión arterial en pacientes obesos. Rev Méd Chile 2005; 133: 511-6.

4. Sjöström L, Narbro K, Sjöström CD, Karason K, Larsson B, Wedel H et al for the Swedish Obese Subjects Study. Effects of Bariatric Surgery on Mortality in Swedish Obese Subjects. N Engl J Med 2007; 357: 741-52.

5. Alvarez-Leite J. Nutrient deficiencies secondary to bariatric surgery. Curr Opin Clin Nutr Metab Care 2004; 7: 569-75.

6. Juhasz-Pocsine K, Rudnicki SA, ARcher RL, Harik SI. Neurologic complications of gastric bypass surgery for morbid obesity. Neurology 2007; 68: 1843-50.

7. BERGER JR. The Neurological Complications of Bariatric Surgery. Arch Neurol 2004; 81: 1185-9.

8. Salas-Salvadó J, García-Lorda P, Cuatrecasas G, BonaDA A, Formiguera X, Del CastiLo D et al. Wernicke's syndrome after bariatric surgery. Clin Nutr 2000; 19: 371-3.

9. Amaral JF, Thompson WR, Caldwell MD, Martin HF, Randall HT. Prospective hematologic evaluation of gastric exclusion survey for morbid obesity. Ann Surg 1985; 201: 186-93.

10. Vargas-Ruiz AG, Hernández-Rivera G, Herrera MF. Prevalence of Iron, Folate, and Vitamin B12 Deficiency Anemia After Laparoscopic Roux-en-Y Gastric Bypass. Obes Surg 2008; 18: 288-93.

11. Csendes A, Burdiles P, Papapietro K, Díaz JC, Maluenda F, Burgos A, Rojas J. Results of gastric bypass plus resection of the distal excluded gastric segment in patients with morbid obesity. J Gastrointest Surg 2005; 9: 121-31.

12. Coates PS, Fernstrom JD, Fernstrom MH, Schauer PR, GREENSPAN SL. Gastric bypass surgery for morbid pacientes producto de la cirugía, especialmente entre los más pobres. No obstante estos resultados debiesen poner una señal de alerta en cuanto a evaluar niveles de vitamina $\mathrm{D}$ en obesos tanto operados como no operados y monitorizar estrechamente la suplementación, considerando que las alteraciones son potencialmente corregibles sólo suplementando con dosis altas de vitamina D.

obesity leads to an increase in bone turnover and a decrease in bone mass. J Clin Endocrinol Metab 2004; 89: 1061-5.

13. Goode LR, Brolin RE, Chowdhury HA, Shapses SA. Bone and gastric bypass surgery: effects of dietary calcium and vitamin D. Obes Res 2004; 12: 40-7.

14. Rajesh R, Panchwagh DO, Graham T. Severe protein calorie malnutrition as a late complication post bariatric surgery: a case report. The Am J Gastroent 2001; 96: S231.

15. Folope V, CoËFfier M, DÉchelotte P. Nutritional deficiencies associated with bariatric surgery. Gastroenterol Clin Biol 2007; 31: 369-77.

16. Carrasco F, Kiatassen J, Papapietro P, Reyes E, Rodríguez L, Csendes A ET al. Propuesta y fundamentos para una norma de manejo quirúrgico del paciente obeso. Año 2004. Rev Méd Chile 2005; 133: 699-706.

17. KUSHNER R. Managing the obese patient after bariatric surgery: a case report of severe malnutrition and review of the literature. J Parent Enter Nut 2000; 24: 126-32.

17. Flum DR, Salem L, Elrod JA, Delunger eP, Cheadle A, CHAN L. Early mortality among Medicare beneficiaries undergoing bariatric surgical procedures. JAMA 2005; 294: 1903-8.

18. Livingston EH, Langert J. The Impact of Age and Medicare Status on Bariatric Surgical Outcomes. Arch Surg 2006; 141: 1115-20.

20. Serra Majem LL, Ribas Barba L. Recordatorio de 24 horas. En: Nutrición y Salud Pública. Métodos, bases científicas y aplicaciones. Ed. Serra López Vioque J. España 1997.

21. FAO. Application of brief scale dietary surveys. Field Manual. Nutrition and Agriculture № 5, 1992.

22. Bunout D, Barrera G, Leiva L, Gattas V, de la Maza MP, Avendaño M, Hirsch S. Effects of vitamin D supplementation and exercise training on physical performance in Chilean vitamin D deficient elderly subjects. Exp Gerontol 2006; 41: 746-52.

23. ShaH M, Simha V, GARg A. Long-Term Impact of Bariatric Surgery on Body Weight, Comorbidities, and Nutritional Status. J Clin Endocrinol Metab 2006; 91: 4223-31.

24. Hertrampf E, Cortés F. Folic acid fortification of wheat flour: Chile. Nutr Rev 2004; 62: S44-8. 
25. Hirsch S, De la Maza P, Barrera G, Gattás V, Petermann M, Bunout D. The Chilean flour folic acid fortification program reduces serum homocysteine levels and masks vitamin B-12 deficiency in elderly people. J Nutr 2002; 132: 289-91.

26. Duran de Campos C, Dalcanale L, Pajecki D, Garrido AB JR, HalPERn A. Calcium Intake and Metabolic Bone Disease after Eight Years of Roux-en-Y Gastric Bypass. Obes Surg 2008; 18: 386-90.

27. Goldner WS, Stoner JA, Thompson J, Taylor K, Larson L, ERICKSON J ET AL. Prevalence of vitamin d insufficiency and deficiency in morbidly obese patients: a comparison with non-obese controls. Obes Surg 2008; 18: 145-50.

28. Ybarra J, Sánchez-Hernández J, Gich I, De Leiva A, Rus X, RodRÍGUEZ-EsPINOSA J ET AL. Unchanged hypovitaminosis D and secondary hyperparathyroidism in morbid obesity after bariatric surgery. Obes Surg 2005; 15: 330-5.

29. Vilarrasa N, Maravall J, Estepa A, Sánchez R, Masdevall C, NAVARRO MA ET AL. Low 25-hydroxyvitamin D concentrations in obese women: their clinical significance and relationship with anthropometric and body composition variables. J Endocrinol Invest 2007; 30: 653-8.

30. HeAney RP. Functional indices of vitamin D status and ramifications of vitamina D deficiency. Am J Clin Nutr 2004; 80: 1706S-9S.

31. Bunout D, Barrera G, Leiva L, Gattas V, De la Maza MP, Avendaño M ET AL. Effects of vitamin D supplementation and exercise training on physical performance in Chilean vitamin D deficient elderly subjects. Exp Gerontol 2006; 41: 746-52.
32. Bunout D, Barrera G, Leiva L, Gattas V, De la Maza MP, HASCHKE F ET AL. Effect of a nutritional supplementation on bone health in Chilean elderly subjects with femoral osteoporosis. J Am Coll Nutr 2006; 25: 170-7.

33. Glatzle J, Piert M, Meile T, Besenthal I, Schafer JF, KonigsRainer A ET al. Prevalence of vertebral alterations and the effects of calcium and vitamin D supplementation on calcium metabolism and bone mineral density after gastrectomy. Br J Surg 2005; 92: 579-85.

34. Bisbalue S, Buus S, Lund B, Hessov I. Food intake and nutritional status after gastrectomy. Hum Nutr Clin Nutr 1986; 40: 301-8.

35. Schweitzer DH. Mineral metabolism and bone disease after bariatric surgery and ways to optimize bone health. Obes Surg 2007; 17: 1510-16.

36. Compher CW, BADELINo KO, Boulata JI. Vitamin D and the bariatric surgical patient: a review. Obes Surg 2008; 18: 220-4.

37. Carlin AM, Rao DS, Yager KM, Genaw JA, Parikh NJ, SzYMANSKI W. Effect of gastric bypass surgery on vitamin D nutritional status. Surg Obes Relat Dis 2006; 2: 638-42.

38. Giusti V, Gasteyger C, Suter M, Heraief E, Gailard Rc, BURCKHARDT P. Gastric banding induces negative bone remodelling in the absence of secondary hyperparathyroidism: potential role of serum C telopeptides for follow-up. Int J Obes (Lond) 2005; 29: 1429-35.

39. Flancbaum L, Belsley S, Drake V, Colarusso T, Tayler E. Preoperative nutritional status of patients undergoing Roux-en-Y gastric bypass for morbid obesity. J Gastrointest Surg 2006; 10: 1033-7. 